\title{
GI-MS45-05 | Using Crystal Structures to Guide Pharmaceutical Drug Substance DeVelopment
}

Agnew, Lauren (AstraZeneca, Macclesfield, GBR)

The use of crystal structures to understand the behaviour of compounds in the development of drug substance and drug product processes is becoming commonplace in industry. This talk will look at how the use of crystal structures and the learning from the interrogation of their structural features has laid the foundation blocks for developing compounds and launching drugs to the market.

An outline will be given to show how industrial scientists use a variety of tools to build up an understanding of the solid form. It will then show how this knowledge is communicated to wider project teams of non-specialists to impact manufacturing processes and crystallisation development, formulation design, particle sizing, setting of storage conditions, supporting regulatory submissions and manufacturability of the drug substance.

A number of case studies will be shown throughout the development of drug products to show how the findings from crystal structure analysis have been successfully communicated to project teams to help solve and explain problems in the manufacture of a number of pharmaceutical compounds. 